\title{
NON-LINEARLY RESTORING PERFORMANCE OF CATENARY MOORING-LINE UNDER CONSIDERATION OF ITS DYNAMIC BEHAVIORS
}

\author{
Shuangxi Guo \\ a. AVIC Composite Corporation LTD, National \\ Key Laboratory of Advanced Composites \\ Beijing 100095, China \\ b. Key Laboratory of Mechanics in Fluid Solid \\ Coupling System, Institute of Mechanics, \\ Chinese Academy of Sciences \\ Beijing 100190, China

\section{Weimin Chen} \\ a. Key Laboratory of Mechanics in Fluid Solid \\ Coupling System, Institute of Mechanics, \\ Chinese Academy of Sciences \\ Beijing 100190, China \\ b. School of Engineering Science, University of \\ Chinese Academy of Sciences \\ Beijing 100049, China \\ wmchen@imech.ac.cn
}

\section{ABSTRACT}

Floating bodies such as oil rig/production platform and wind turbine in ocean need to be fixed or controlled at expected position by its supporting system which includes tension tendon and catenary mooring-line. Recently, the later one, catenary mooring-line, is increasingly used in deeper water due to its lower cost and easier installment. As the floating platform are developed toward deeper water depth, the length of the mooring-line become larger and consequently the dynamic behaviors such as the structural inertia and hydrodynamic inertia/damping of the mooring-line become more obvious.

In this paper, the dynamic behaviors of the mooring-line are considered, and compared with the traditional quasi-static method where only the static restoring force is involved, so as to comprehensively examine the non-linearly restoring performance of catenary mooring-lines. Firstly, the nonlinear dynamic model of the mooring system is developed based on our $3 d$ dynamic catenary equations along with the modified finite element simulations. Compared with the static restoring force, essentially depending on structural gravity and overall shape based on static catenary theory, the dynamic restoring

\author{
Yilun Li \\ Sino-French Engineering School, Beijing \\ University of Aeronautics and Astronautics \\ Beijing 100191, China
}

\author{
Yiqin $\mathrm{Fu}$ \\ Key Laboratory of Mechanics in Fluid Solid \\ Coupling System, Institute of Mechanics, \\ Chinese Academy of Sciences \\ Beijing 100190, China
}

force is analyzed based on our $3 d$ curved flexible beam approach where the structural curvature changes with its spatial position and time in terms of vector equations. In our modified finite element simulations, the rotation degree of freedom between neighboring beam elements is released and bending stiffness of individual element is set to be zero, and the statically original shape and top tension according to the traditional static catenary theory are used as the initial conditions. Moreover, the hydrodynamic force is loaded as depending on structural motion.

Based on our numerical simulations, the influences of the amplitude and frequency of the catenary's top-end motion, along with the structural parameters (including the mass density and initial tension ratio), on mooring line's temporalspatial evolution of displacement and dynamic tension are studied. Also, the slack-taut phenomenon caused by structural hydrodynamic inertia and damping are presented. Our results show: 1) Generally, the displacement distribution along the mooring-line is characterized as a stable stand wave. The additional part of restoring tension due to the dynamic effects is up to $20 \%$ of the quasi-static method, and the tension amplitude

${ }^{*}$ Corresponding author, Email: wmchen@imech.ac.cn 
difference (between the maximum tension and minimum tension) is around three times of the quasi-static value. Particularly, as the mooring-line becomes slack, the response is characterized as travelling wave, the maximum tension amplitude is up to 9 times of the static method. 2) As the amplitude/frequency of the catenary's top-end motion increases, the value of catenary displacement firstly drops and then rises. The displacement distribution along catenary length changes with the motion of top end. Interestingly, the maximum displacements occur at the middle point of the catenary for case of surge while the maximum displacement moves up along the catenary as the top end motion gets larger for case of heave. 3) The magnification factor of top tension drops with increase of mooring-line mass density but rises with the increase of the initial tension ratio. It is also noted the velocity amplitude at higher frequency in the velocity spectrum may increase as the top end motion increases.

Keywords: dynamic response; catenary mooring-line; dynamic behavior; slack-taut

\section{INTRODUCTION}

In recent years, more and more wind energy industries have been developing towards ocean area because of the advantages of ocean wind resource, e.g. higher wind speed, more steady wind field and lower level of noise to the residents onshore. Generally, the floating wind turbine, mostly being used in ocean area and somewhat similar with the ocean oil/gas exploitation platform, needs to be positionally controlled or fixed in a certain range by its supporting system. More specifically, the translating and rotational motions of the floating body should be no less the threshold value, and the economic cost of mooringlines is supposed to be as low as possible under the condition of required structure strength. Therefore, accurate analysis of mooring-lines' restoring performance, along with its dynamic response under current loads, and the floating body motion is one of important, and also pretty challenging, issues, which is essentially needed to provide necessary data base for strength and stability design of the mooring-lines and whole floating system. As we know, the supporting structures, principally designed for the floating body, of the wind turbine operating in the ocean area are quite different from its counterpart, actually being fixed structure, of onshore wind turbine. By now, according to the types of floating bodies and water depth, these supporting systems mainly include three types[1]: catenary mooring-lines, tendon lines and vertical tension legs. The first one among the three ones, catenary mooring-line, is increasingly used due to its lower economic cost and easier installment implementation.

Most fruitful researches[2-7] of the mooring-line are about its restoring performance and floating body stability. Among these, the mostly popular approach is essentially a kind of quasi-static method where the mooring-line is simplified as a static spring and then the statically restoring force is mainly considered there. For examples, Mavrakos[4] analyzed the top tension and movement of mooring-line by the numerical simulations, and Papazoglou[5] presented the main factors which may influence restoring performance of a catenary. Van Den Boom[6] found that the nonlinearities coming from the geometry, elastic deformation and acting loads can significantly enlarge top tension. Gobat[7] studied the influences of floating vessel on mooring-line tension by using an empirical model.

It is noted that as a flexible body with large displacement under complex action of ambient fluid load, the behaviors of mooring-line often exhibit strong nonlinear characteristics which introduce significant challenge to the calculation of its restoring performance. Qiao[8] presented the static restoring force of a catenary, made up of three wire ropes and chains, with different buoyancy unit weights and structural properties based on classic static catenary theory and piecewise extrapolation; Zhang[9] provided a simplified method to study the static horizontal tension of a mooring-line with multicomponents by using the golden-section method and solving the catenary equation, which is particularly suitable for the computing iterations of static mooring systems.

However, as water depth and structural length increase, the dynamic characteristics of mooring-line become more significant, which may consequently change top tension and, even, may introduce transiently large snap tension due to mooring-line slack-taut. Based on an simplified model where the two structural ends were assumed at same level and the initial catenary displacement is small, Vassalos[10] gave the dynamic responses of a catenary mooring-line, then he examined the mooring-line slack and snap tension by using a centered-mass model[11]. Chen[12] calculated the dynamic response of a system including a spar and its mooring-lines based on a linear coupling approach. He pointed out that if the inertial and damping forces of the mooring-line are involved during dynamic response, the top tension would get larger. Zhang[13] experimentally investigated the tension of a slacktaut case and found the snap tension was 5 times of the static one due to slack.

To summarize, two kinds of methods, i.e. lumped-mass method and flexible-bar method, were used to involve the dynamic behavior, mainly the structural inertia force, of catenary. The lumped-mass model where a series of concentrated-mass points connected by linear springs can consider the inertial force of the structure and the added mass coming from the fluid, while the values of spring stiffness are estimated, before the calculation, according to static catenary theory and the structural stiffness and motion are assumed to be linear. For another method, the flexible-bar model, the nonlinear dynamic equations of curved flexible bar are build and to be numerically solved so as to model nonlinear structural stiffness and to get large displacement of catenary. Since the nonlinearity of the equations which become more difficult to be solved, certain assumption is used sometimes. Given an ideal assumption of the two ends of catenary being at same level, Zhang[14] studied the nonlinear dynamic response of a catenary. Still, the dynamic behaviors of mooring line need further research to model a practical catenary with less 
assumptions, and few results of the extreme situations such as slack-taut based on the current approaches are seen.

Given above mentions, in this study we develop a modified flexible-bar model combining with the FEM simulations so as to comprehensively examine the non-linearly restoring performance of catenary mooring-lines under consideration of its dynamic behaviors. By our model, the dynamic response and vibration wave propagation can be solved in time domain to present the temporal-spatial evolution of dynamic tension and displacement along catenary length, and, moreover, the slacktaut phenomenon along with its snap tension during the dynamic response is examined. In section 2, the nonlinear dynamic model of the mooring system is developed, where the structural curvature changes with time and its spatial position in terms of vector equations. And, the hydrodynamic force acted by ambient fluid field on the mooring-line is loaded as depending on structural motion. In sections 3 and 4, the influences of topend motion, e.g. its amplitude and frequency, and the mooringline's structural properties, e.g. mass density and initial shape, on mooring line's restoring performance including its dynamic displacement and tension are studied respectively, as well as the slack-taut phenomenon during the dynamic response.

\section{DYNAMIC GOVERNING EQUATIONS OF A MOVING CATENARY MOORING-LINE AND FEM APPROACH}

According to the classical catenary theory which is pretty popular and frequently used to a catenary mooring-line, the top tension (static restoring force), essentially depending on structural gravity and overall shape, can be given by solving the static equations[8]. In other words, only the static restoring force of the mooring system is involved. Here, in order to consider the nonlinear geometry, structural inertial and damping and the fluid drag forces, we develop the dynamic governing equations. And in this case, the dynamic restoring force can be analyzed based on our $3 \mathrm{~d}$ curved flexible beam approach where the structural curvature changes with its spatial position and time in terms of vector equations. For a $3 \mathrm{~d}$ catenary (see Fig.1), compared with the previous $2 \mathrm{~d}$ static model, the dynamic equations includes some nonlinear terms, that makes the analysis of dynamic response strong nonlinear and suitable to get more reasonable response of a moving catenary[15-18]. The governing equations of dynamics in terms of vectors $[19,20]$ can be written as:

$$
\begin{gathered}
\vec{F}^{\prime}+\vec{q}=\rho A \ddot{\vec{r}} \\
\vec{M}^{\prime}+\vec{r}^{\prime} \times \vec{F}+\vec{m}=0
\end{gathered}
$$

where $\boldsymbol{F}$ and $\boldsymbol{M}$ are respectively the total force and moment of the catenary. $\boldsymbol{q}$ and $\boldsymbol{m}$ are respectively the outer force and moment acted on per unit length of the catenary. $\rho$ and $\boldsymbol{A}$ are structural mass density and area respectively. $r$ represents the position vector.

Then the expression of the bending moment and curvature is:

$$
\vec{M}=\vec{r}^{\prime} \times\left(B \vec{r}^{\prime \prime}\right)+H \vec{r}^{\prime}
$$

where $\boldsymbol{B}$ is the structural stiffness and $\boldsymbol{H}$ is the torsion moment. Substituting Eq.(3) into (2), we have:

$$
\vec{r}^{\prime} \times\left[\left(B \vec{r}^{\prime \prime}\right)^{\prime}+\bar{F}\right]+H^{\prime} \bar{r}^{\prime}+H \bar{r}^{\prime \prime}+\vec{m}=0
$$

and:

$$
H^{\prime}+\vec{m} \vec{r}^{\prime}=0
$$

where $\vec{m}$ is the averaged rotation moment whose value will be zero if $\vec{m} \vec{r}=0$, then $\boldsymbol{H}=0$, that means the torsion moment is independent on the structural arc length. Generally, the rotation moment can be neglected, or the values of both $\boldsymbol{H}$ and $\vec{m}$ are zero. Then Eq.(4) can be rewritten as:

$$
\vec{r}^{\prime} \times\left[\left(B \vec{r}^{\prime \prime}\right)^{\prime}+\dot{F}\right]=0
$$

or:

$$
\vec{F}=-\left(B \bar{r}^{\prime \prime}\right)^{\prime}+\lambda \bar{r}^{\prime}
$$

Substituting Eq. (7) into (1) will yield:

$$
-\left(B \vec{r}^{\prime \prime}\right)^{\prime \prime}+\left(\lambda \vec{r}^{\prime}\right)^{\prime}+\vec{q}=\rho A \ddot{\vec{r}}
$$

and the deformation equation is:

$$
\vec{r}^{\prime} \cdot \vec{r}^{\prime}=(1+\varepsilon)^{2}
$$

where $\varepsilon$ is the strain of the catenary. If the value of the bending moment in Eq.(7) is zero, we will have the dynamic equation of a catenary of which the external loads include the gravity, buoyancy and hydrodynamic forces.

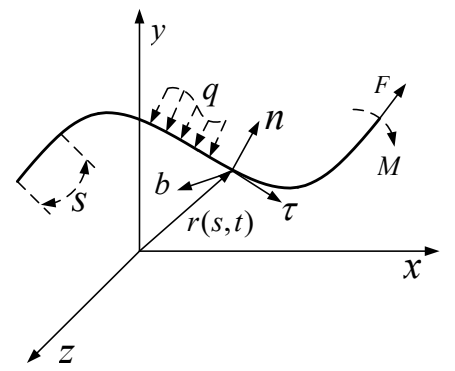

(a)

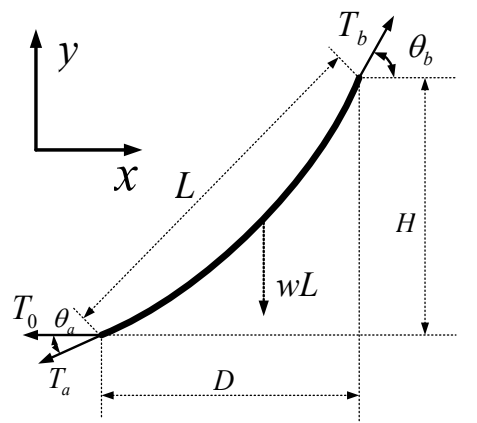

(b)

FIGURE 1. 3D CATENARY MOORING LINE (a) THE ELEMENT OF 3D FLEXIBLE MOORING-LINE (b) SCHEMATIC DIAGRAM OF THE WHOLE CATENARY IN STATIC BALENCE

Generally, the hydrodynamic force acted on per unit structure length can be expressed by the Morison formula [4,12] as:

$f=\frac{1}{2} C_{D} \rho D|V-\dot{u}|(V-\dot{u})+\mathrm{C}_{A} \frac{\pi D^{2}}{4} \rho(\dot{V}-\ddot{u})+\frac{\pi D^{2}}{4} \rho \dot{V}$

where $D$ and $u$ are the structural diameter and displacement respectively. $V$ is the fluid velocity. Combing Eqs.(8), (9) and 
(10), we have a group of nonlinear equations of which direct solution could not be gotten theoretically. Here, a numerical simulation based on FEM is used to solve the dynamic equations.

The catenary is uniformly divided into $N$ elements which are two-node Euler beam element (Craig 1981). For representativeness and simplicity, only the translation displacement in $\boldsymbol{x}-\boldsymbol{y}$ plane $\left[u_{i}, v_{i}\right], \boldsymbol{i}=1,2, \ldots, \boldsymbol{N}+1(\boldsymbol{N}+1$ is the total number of nodes, and $u_{i}, v_{i}$ indicate the translation displacement in directions along with the $\boldsymbol{x}, \boldsymbol{y}$ axes respectively) and one rotation around $\boldsymbol{z}$ axis $\theta_{i}, \boldsymbol{i}=1,2, \ldots, \boldsymbol{N}+1$, of per node, are considered. The three-order polynomial function is used as the displacement expression of the beam element. Then the governing equation of the structure with many degrees of freedom can be written as follow:

$$
(M+\bar{M}) \ddot{U}+C \dot{U}+K U=F
$$

where $\boldsymbol{M}$ and $\bar{M}$ are respectively the structure mass matrix and the added mass matrix. $\boldsymbol{C}$ is the structure damping matrix. $\boldsymbol{K}$ is the stiffness matrix. $\boldsymbol{F}$ is the hydrodynamic force acted by ambient fluid. $U$ is the displacement vector. For a catenary body, in order to model simultaneously its original catenary shape and the large rotation/translation flexibilities of a vibrating catenary under consideration of its dynamic behavior, in our modified finite element simulations, the rotation motion between two neighboring beam elements is released, or the rotation angles of the two beam elements are no longer consistent with each other at same grid. And, the bending stiffness of every individual element is set to be zero. Subsequently, the system rotational degrees of the freedom $\theta$ would double as $\theta, \theta^{\prime}$ because of the additional rotation angle. The displacement vector of beam element changes from the original form

$$
U_{i}=\left[u_{i}, v_{i}, \theta_{i}, u_{i+1}, v_{i+1}, \theta_{i+1}\right]^{T}
$$

as

$$
U_{i}^{\prime}=\left[u_{i}, v_{i}, \theta_{i}, \theta_{i}^{\prime}, u_{i+1}, v_{i+1}, \theta_{i+1}, \theta_{i+1}^{\prime}\right]^{T}
$$

Then, given the statically indeterminate characteristics along with stronger stiffness singularity of the system, additional constrains or original conditions are required to get the definite solution of the dynamic equation. Here the original shape and top tension based on traditional static catenary theory is used as the definite conditions so as to eliminate the singularity of the stiffness matrix, or to model the real catenary mooring-line.

To run the dynamic response analysis, a direct numerical integration method is used to solve the FEM dynamic equations. The Newmark method is employed here so as to adjust the distribution of the structural acceleration and the nonlinearity of the catenary during the integration range by properly changing the integration parameters. The interpolation functions of the displacement and acceleration are written as:

$$
\begin{aligned}
& \dot{U}_{t+\Delta t}=\dot{U}_{t}+\left[(1-\beta) \ddot{U}_{t}+\beta \ddot{U}_{t+\Delta t}\right] \Delta t \\
& U_{t+\Delta t}=U_{t}+\dot{U}_{t} \Delta t+\left[\left(\frac{1}{2}-\alpha\right) \ddot{U}_{t}+\alpha \ddot{U}_{t+\Delta t}\right] \Delta t^{2}
\end{aligned}
$$

where the values of $\alpha$ and $\beta$ are respectively $1 / 6$ and $1 / 2$ at every time step during the dynamic response.

\section{DYNAMIC RESPONSE OF THE CATENARY BASED ON THE NUMERICAL SIMULATIONS}

The main structural and geometrical parameters of the catenary are listed in Table 1 . The dynamic response of the catenary under motion of FWT (Floating Wind Turbine) is analyzed based on our numerical simulations. The sway of FWT is taken as an excitation, then the displacement, velocity and tension of the catenary are calculated.

TABLE 1. THE MAIN GEOMETRICAL AND MATERIAL PARAMETERS OF THE CATENARY

\begin{tabular}{cc}
\hline Geometrical & Value \\
\hline Length & $800 \mathrm{~m}$ \\
Initial horizontal projection & $706 \mathrm{~m}$ \\
Initial vertical projection & $350 \mathrm{~m}$ \\
Diameter & $0.19 \mathrm{~m}$ \\
Young's modulus & $210 \mathrm{GPa}$ \\
Density & $2513 \mathrm{~kg} / \mathrm{m} 3$ \\
Poisson's ratio & 0.3 \\
\hline
\end{tabular}

\subsection{Dynamic Responses under FWT's Motions}

The typical dynamic response of the catenary for case of a regular motion, i.e. $0.05 \mathrm{~Hz}$ frequency and $4 \mathrm{~m}$ surge amplitude, of the FWT is shown in Fig. 2 and 3. In Fig. 2, the top tension of the quasi-static method is also plotted as a comparison. It is seen that either the value of the peaks or the trough get extremer than, or the gap value between peak and trough gets around 3 times of the quasi-static ones owing to the inertial and damping effects, while the period of dynamic tension is consistent with that of the top FWT's surge. Observing the temporal-spatial evolution of the tension, we noted that the maximum tension always occurs at the top end when the values of tension along catenary length get their own peaks, that means tension change with time all at the same phases. Therefore, we may say the top tension is the key point if one wants to check the strength of a moving catenary.

Additionally, the spectrum plots of the top tension during the dynamic response are presented in Fig. 4 where the value at $0.00 \mathrm{~Hz}$ frequency is corresponding to the quasi-static case. At higher frequencies which are a couple of times of the excitation frequency, there are several tension peaks among which the maximum tension is at the excitation frequency, $0.05 \mathrm{~Hz}$. Most likely, those tension peaks in Fig.4b, at higher frequency ranging from $0.3 \mathrm{~Hz}$ to $1.1 \mathrm{~Hz}$, are responsible for the small tension fluctuation with short periods in Fig.2. Moreover, if comparing the static value (at $0.00 \mathrm{~Hz}$ ) with the additional dynamic values (e.g. at $0.05 \mathrm{~Hz}$ ), the later one is around $30 \%$ of the former one. That means the impact caused by the dynamic behavior of the inertial and damping loads is around $30 \%$ of the total static one, and that is consistent with the time history curves in Fig. 2 where the dynamic peak is around 20\% higher than the static peak. 


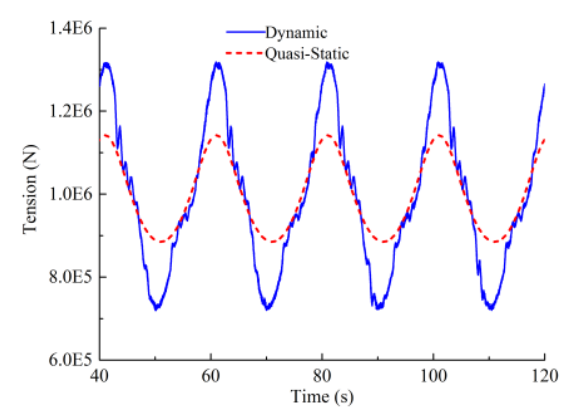

FIGURE 2. TIME HISTORY OF THE TOP TENSION OF THE CATENARY MOORING-LINE

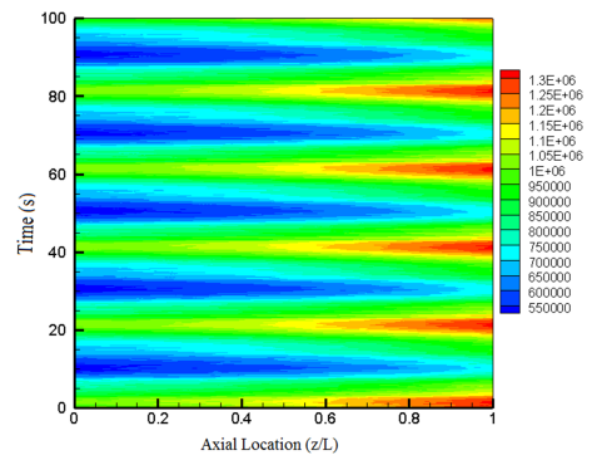

FIGURE 3. TEMPORAL-SPATIAL EVOLUTION OF THE CATENARY TENSION

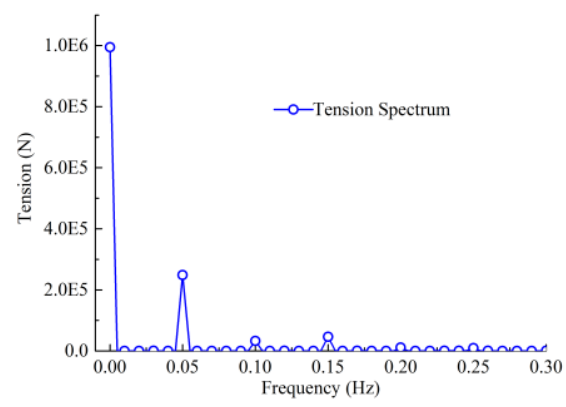

(a)

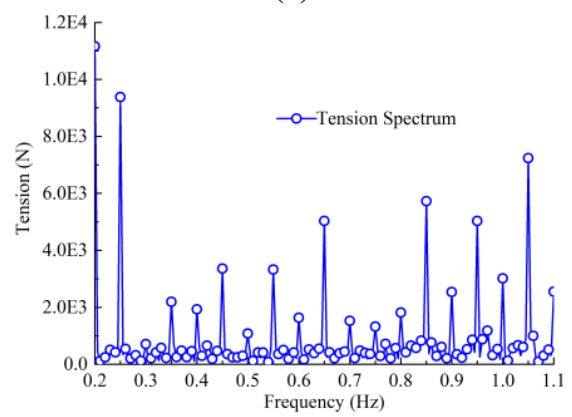

(b)

FIGURE 4. THE SPECTRUM PLOTS OF THE TOP TENSION (a)AT FREQUENCY RANGE 0-0.3HZ (b) AT FREQUENCY RANGE 0.2$1.1 \mathrm{HZ}$

The temporal-spatial evolutions of the displacements are presented in Fig.5. Interestingly, the horizontal displacement has two motion waves, i.e. one is around the middle part of the catenary and another one is close to the top end where the positive wave travels somewhat toward the middle part of catenary. While the vertical displacement has only one stable motion wave. The maximum displacements occur at the top end for case of the horizontal displacement and at middle point for vertical displacement respectively. Generally speaking, the movement of the catenary is characterized as a stable standing wave, or no apparent travelling wave is seen.

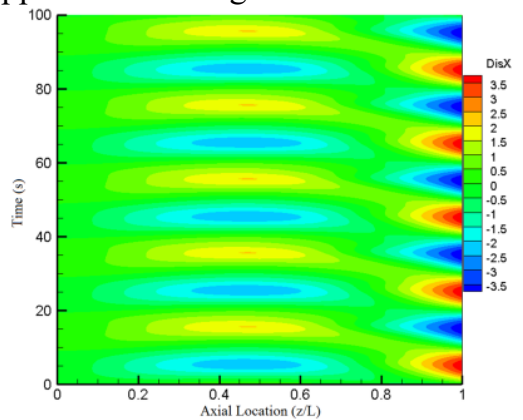

(a)

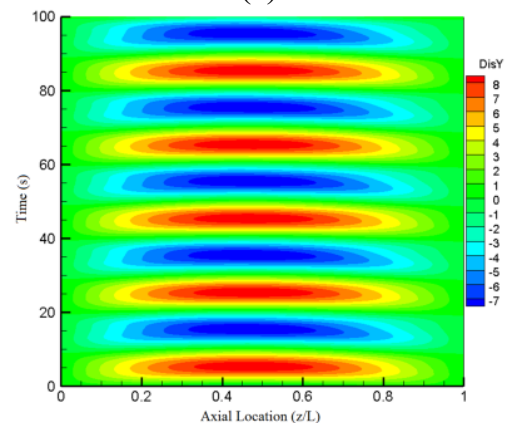

(b)

FIGURE 5. TEMPORAL-SPATIAL EVOLUTION OF THE CATENARY DISPLACEMENT (a) THE HORIZONTAL DISPLACEMENT (b) THE VERTICAL DISPLACEMENT

The plots of the velocity versus displacement of the catenary middle point are presented in Fig.6, which approximately are elliptical. It is seen that both the horizontal and vertical motions are periodic cycles. And, the motion center of the horizontal displacement is somewhat negative to the point with zero value because of the initial static deformation due to the structural gravity of the flexible catenary. Notably, compared with the gradually stable vertical velocity, the horizontal velocity fluctuates a little during a whole motion period probably owing to the nonlinearity of the mooring-line.

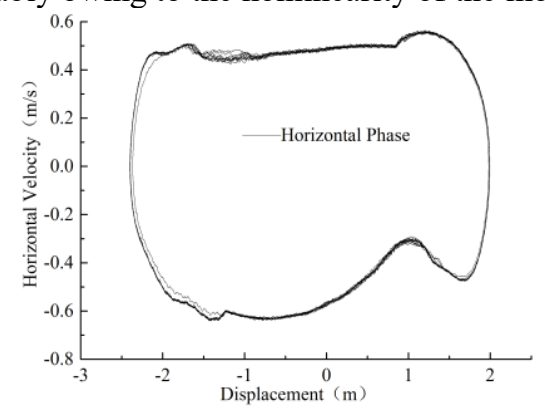

(a) 


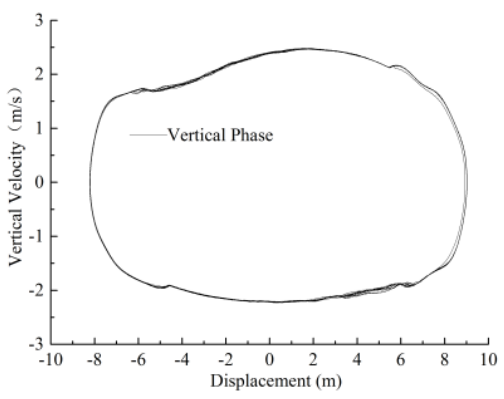

(b)

FIGURE 6. PHASE TRACK OF THE VELOCITY VERSUS DISPLACEMENT OF THE MIDDLE POINT OF THE CATENARY LINE (a) THE TRACK OF THE HORIZONTAL MOTION (b) THE TRACK OF THE VERTICAL MOTION

\subsection{The Slack-Taut Phenomenon and Its Dynamic Tension}

The top tension under the condition of the top movement with $0.1 \mathrm{~Hz}$ frequency and $6 \mathrm{~m}$ amplitude is give in Fig.7. There is an abrupt increase of the dynamic tension while the minimum tension reaches zero value, which means slack-taut happens. In this case, the maximum tension is around three times of the static method and even the peak-trough value is about ten times of the static method. From the temporal-spatial evolution given in Fig. 8, we can see that the maximum tension is at the top end $(\mathrm{z} / \mathrm{L}=1.0)$ of the catenary when slack-taut happens. And, observing the spectrum plot of top tension give in Fig. 9, it is seen that the tension amplitude is much larger than the static method and gradually drops with the increase of the frequency mainly owing to the slack-taut phenomenon. For examples, compared with Fig. 4, the tension amplitude at excitation frequency is higher than the static method by about $30 \%$ and the tension amplitude at twice excitation frequency is about $50 \%$ of the static method.

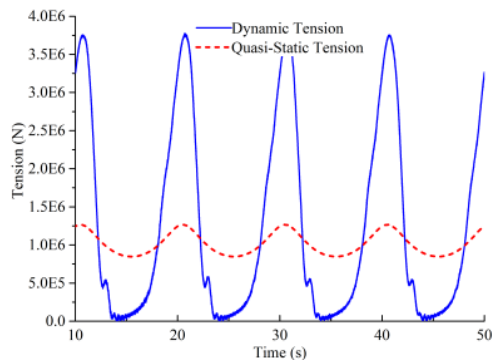

FIGURE 7. THE TIME HISTORY OF THE TOP TENSION DURING SLACK-TAUT CONDITION

It is noted that the temporal-spatial evolutions of the displacements and velocity change significantly, see in Fig.10 and 11. Obvious travelling wave can be seen there in horizontal displacement, especially in positive displacement (red color in Fig.10a), though there are two motion waves which is similar with Fig. 5. Additionally, the original one stable motion wave of vertical displacement split into two shorter waves of negative displacement. The vertical maximum values respectively occur at the middle point for positive displacement and at the top/bottom ends for negative, which is quite different from Fig. $5 b$.

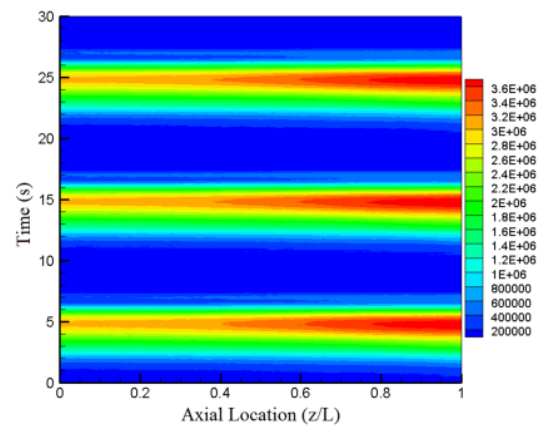

FIGURE 8. THE TEMPORAL-SPATIAL EVOLUTION OF TENSION DURING SLACK-TAUT CONDITION

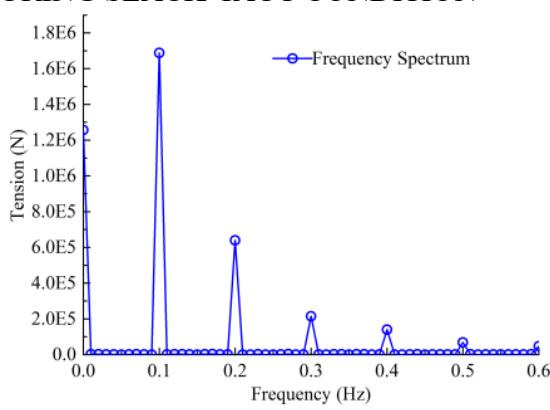

(a)

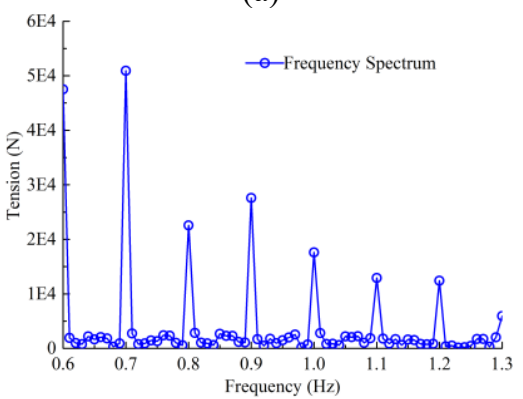

(b)

FIGURE 9. THE SPECTRUM PLOTS OF THE TOP TENSION (a) AT FREQUENCY RANGE 0-0.6HZ (b) AT FREQUENCY RANGE $0.6-1.3 \mathrm{HZ}$

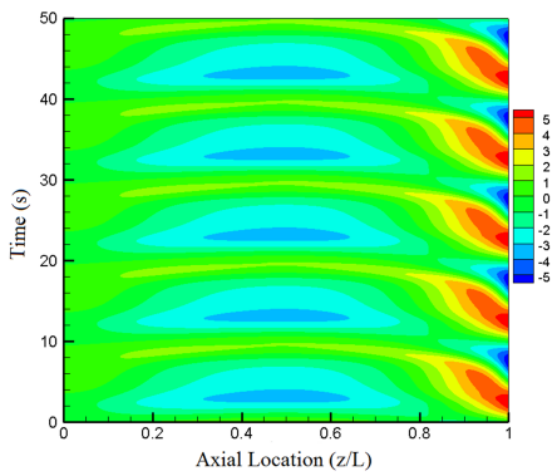

(a) 


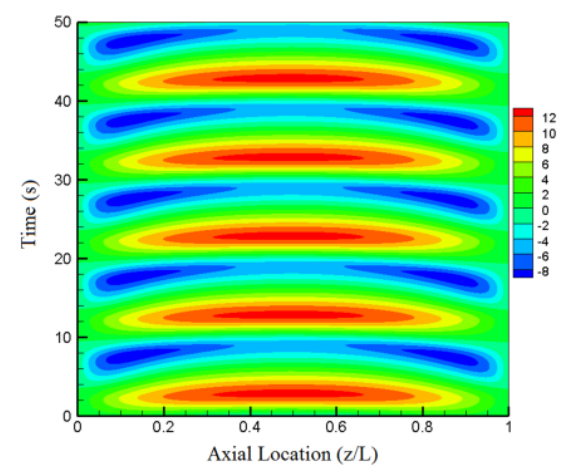

(b)

FIGURE 10. TEMPORAL-SPATIAL EVOLUTION OF THE CATENARY DISPLACEMENT (a) THE HORIZONTAL DISPLACEMENT (b) THE VERTICAL DISPLACEMENT

If concerning the plots of the velocity versus displacement of the catenary middle point, see Fig.11, the motion center of the horizontal displacement moves toward much negative to the point zero, and there is a little horizontal velocity fluctuation as the displacement changes from negative to positive value to reach its maximum value. Interestingly, Fig.11b indicates that the vertical velocity almost keeps at a constant value, i.e. the minimum velocity, as the displacement change from its positive peak to negative trough. That means owing to the additional dynamic behavior of the catenary, the structural initial/damping forces and fluid drag force can balance the structural gravity which is supposed to principally cause the structural tension, and consequently, slack would happen if no tension is caused.

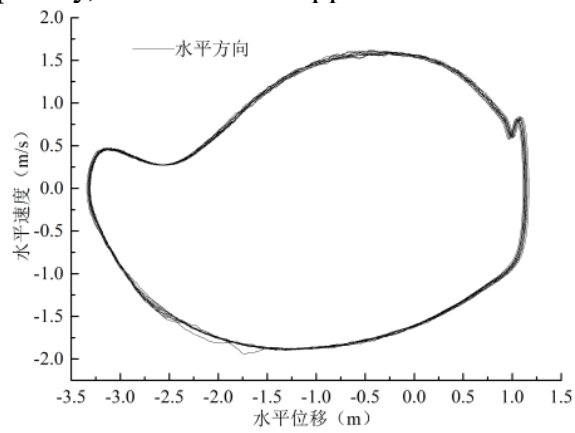

(a)

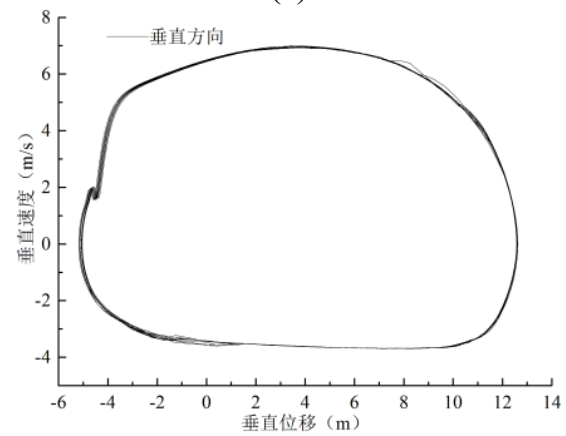

(b)

FIGURE 11. PHASE TRACK OF THE VELOCITY VERSUS DISPLACEMENT OF THE MIDDLE POINT DURING SLACK CONDITION (a) THE TRACK OF THE HORIZONTAL MITION (b) THE TRACK OF THE VERTICAL MOTION

\section{INFLUENCES OF TOP-END MOVEMENT AND STRUCTURAL PROPERTIES ON THE DYNAMIC RESPONSE}

There are some factors, such as the movement and initial position of top-end and the material parameters of the catenary, which may influence the final response of the mooring-line. Here the impacts of the motion of top-end and the structural properties, such as the initial shape and mass, on the dynamic response of the catenary are examined, because the mass (the material density or that of the connected buoyancy) or shape may directly change the tension and then the dynamic response.

\subsection{Influences of the Top-End Motion on the Dynamic Response}

The RMS (root-mean square, referred as RMS later) displacements, normalized by the top-end amplitude, are presented in in Fig. 12, at $0.1 \mathrm{~Hz}$ frequency and $3-10 \mathrm{~m}$ amplitude of top-end surge (Fig. 12ab), and at $0.1 \mathrm{~Hz}$ frequency and $3-13 \mathrm{~m}$ amplitude of top-end surge (Fig. 12cd). It is seen that the maximum displacement is around the middle of the catenary, and it drops first then rises with the increase of top-end amplitude. As the amplitude gets larger, i.e. more than $6 \mathrm{~m}$, there are shorter waves at each end of the catenary, which becomes more apparent and could cause locally higher stress. Interestingly, the maximum displacement occurs at the middle point of the catenary for case of surge while the maximum displacement moves up along the catenary as the top end motion gets larger for case of heave.

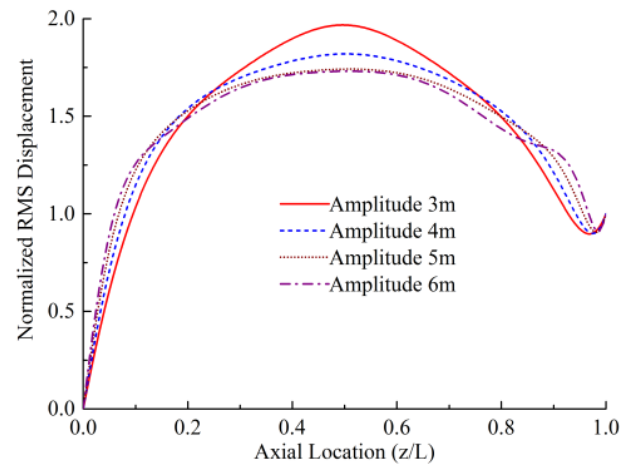

(a)

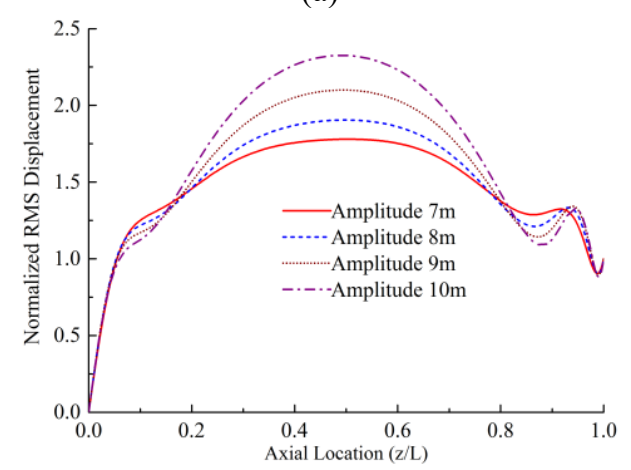

(b) 


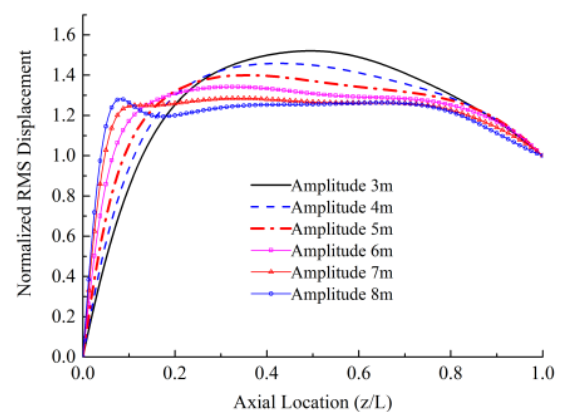

(c)

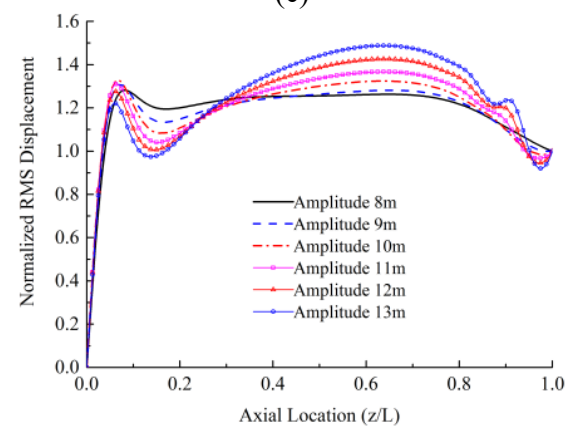

(d)

FIGURE 12. NORMALIZED RMS DISPLACEMENT (a) AT THE TOP-END SURGE AMPLITUDE 3-6m (b) AT THE TOP-END AMPLITUDE SURGE 7-10m (c) AT THE TOP-END HEAVE AMPLITUDE 3-7m (d) AT THE TOP-END AMPLITUDE HEAVE 7$13 \mathrm{~m}$

Fig. 13 presents the time history of middle point velocity at respectively $6 \mathrm{~m}$ and $9 \mathrm{~m}$ top-end amplitude, where the velocity gets larger as the top-end amplitude increases. For the vertical velocity, see Fig.13b, during the trough the velocity stably stay at the minimum value, that means the acceleration is almost zero, or the slack is happening.

While, for the horizontal velocity, fluctuation can be seen around the peak. The velocity spectrum, see Fig. 14, shows that the velocity amplitude at higher frequency becomes larger as the top-end amplitude increases, e.g. the amplitude at twice excitation frequency is large than that at the excitation frequency for case of $9 \mathrm{~m}$ top-end amplitude. It is also noted that the tension amplitude at higher frequency becomes larger as the top-end amplitude increases, see Fig.14c. As we know, the higher tension at higher frequency should be paid more attention because it is much crucial to fatigue life of catenary during structure safety assessment.

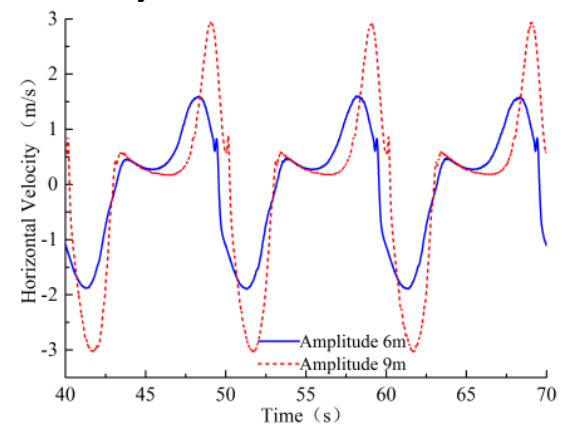

(a)

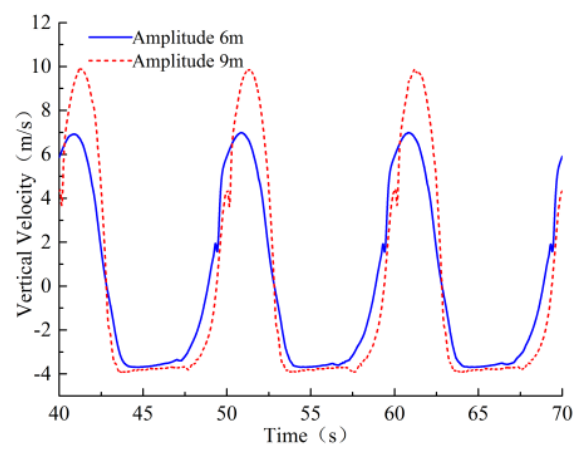

(b)

FIGURE 13. THE TIME HISTORY OF MIDDLE POINT VELOCITY OF MOORING-LINE (a) THE HORIZONTAL VELOCITY (b) THE VERTICAL VELOCITY

In fact, the catenary tension is one of important factors that should be considered during mooring-ling design, because it can influence mooring-line structure strength and also motion stability of floating system. The maximum/minimum tensions at different amplitudes and frequencies of top-end movement are presented in Fig. 15 and 16 respectively, and the tensions of quasi-static method are also plotted there as a comparison. Generally, as the top-end motion gets larger, the maximum tension gradually increases, up to around 9 times (in Fig. 16) of the quasi-static one, but the minimum tension gradually drops to zero till the amplitude/frequency is more than $6 \mathrm{~m} / 0.10 \mathrm{~Hz}$.

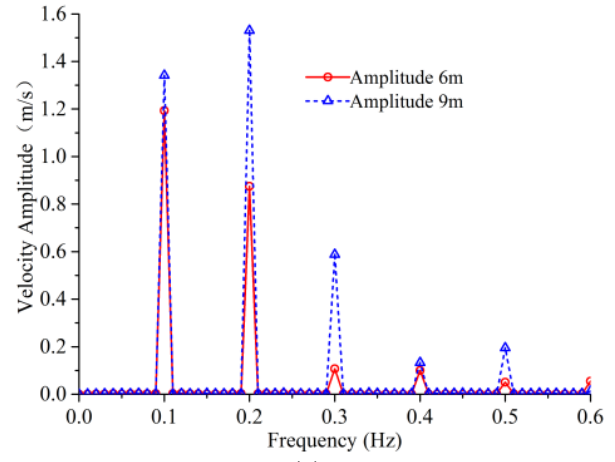

(a)

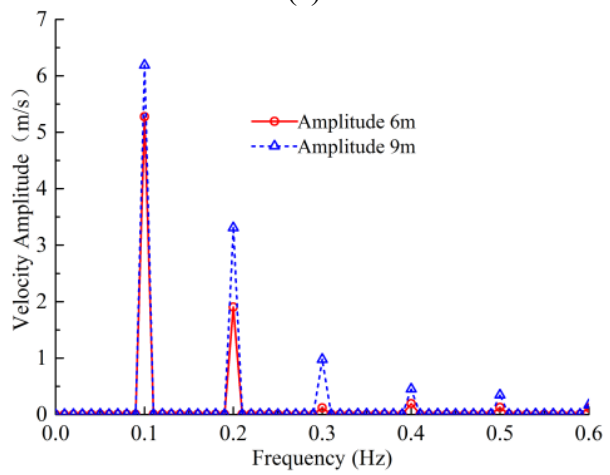

(b) 


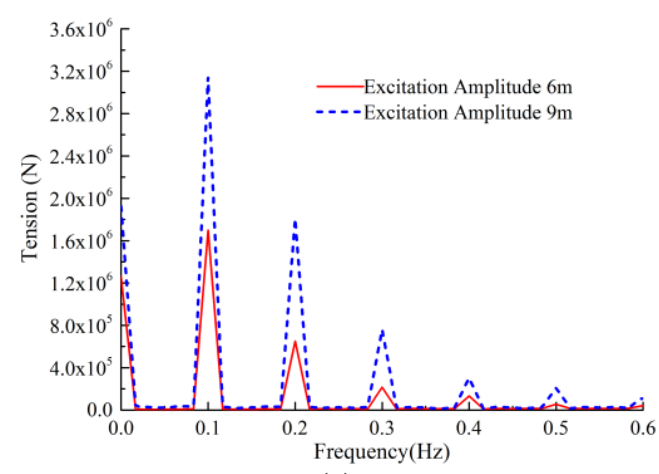

(c)

FIGURE 14. AMPLITUDE-FREQUENCY OF THE VELOCITY AND TENSION (a) THE HORIZONTAL VELOCITY SPECTRUM (b) THE VERTICAL VELOCITY SPECTRUM (c) THE TENSION SPECTRUM

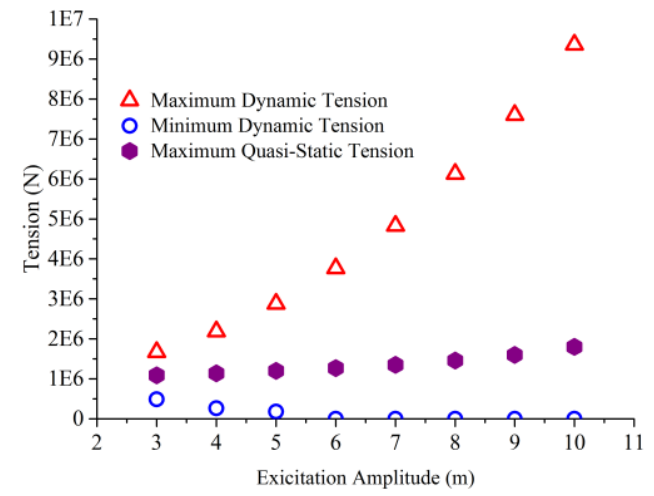

FIGURE 15. THE MAXIMUM/MINIMUM TENSION VERSUS THE TOP-END AMPLITUDE AT $0.10 \mathrm{~Hz}$

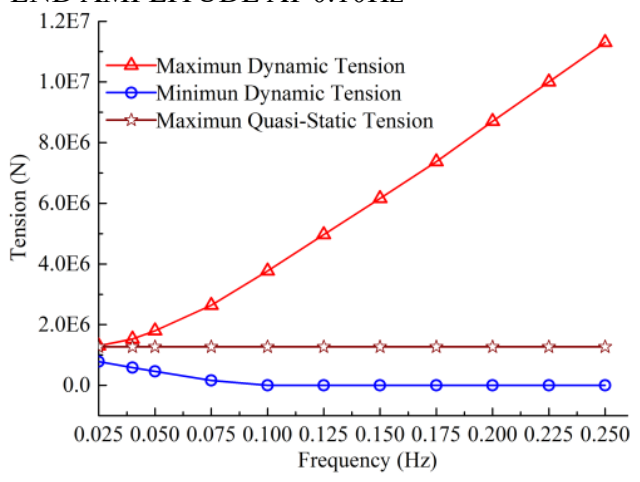

FIGURE 16. THE MAXIMUM/MINIMUM TENSION VERSUS THE TOP-END FREQUENCY AT 6m AMPLITUDE

\subsection{Influences of Structural Properties of the Catenary on the Dynamic Response}

The values of all selected density range as $8000 \mathrm{~kg} / \mathrm{m} 3$; $10,000 \mathrm{~kg} / \mathrm{m} 3 ; 11,200 \mathrm{~kg} / \mathrm{m} 3 ; 12,000 \mathrm{~kg} / \mathrm{m} 3$ and $14,000 \mathrm{~kg} / \mathrm{m} 3$.

The RMS displacements along the catenary length are plotted in Fig. 17 at FWT's movement of $0.1 \mathrm{~Hz}$ frequency along with $6 \mathrm{~m}$ and $9 \mathrm{~m}$ amplitude respectively. As mass density gets smaller, the displacement gets larger while the slack gets more likely to happen. And, the short wave near the top end becomes more apparent of which wave length decreases but the wave amplitude increases. It is also noted that, for case of 6m FWT's amplitude, the maximum displacement (almost close to the middle point of the catenary) rises as the mass density gets larger while, for case of 9m FWT's amplitude, the maximum displacement drops. The reason for this might be the hydrodynamic drag which is proportional to the square of structural velocity. As the mass gets smaller (then the structural velocity gets larger), the damping of hydrodynamic drag to the structure movement becomes more significant than the effect of mass decrease which originally make the movement of catenary rise. Figure 18 shows the tension amplification ratio of the top tension at different mass densities, where the tension amplification rises with the increase of FWT's amplitude or the decrease of mass density and the curves gets steeper with the decrease of mass density.

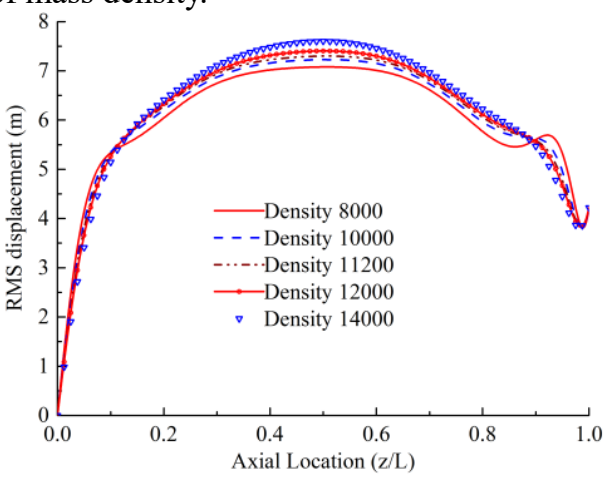

(a)

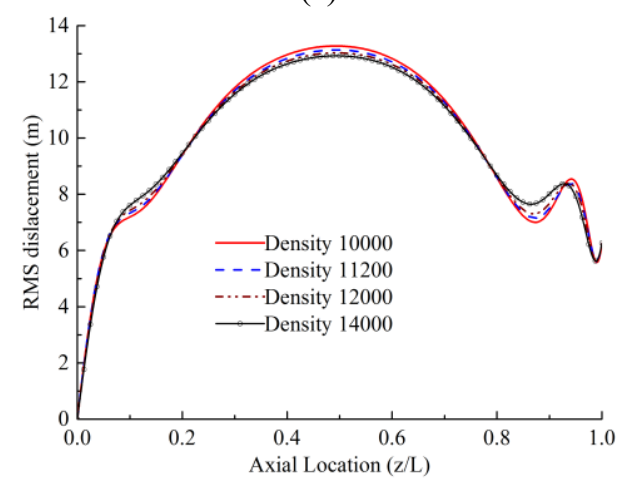

(b)

FIGURE 17. RMS DISPLACEMENT (a) AT 6m FWT AMPLITUDE (b) AT 9m FWT AMPLITUDE

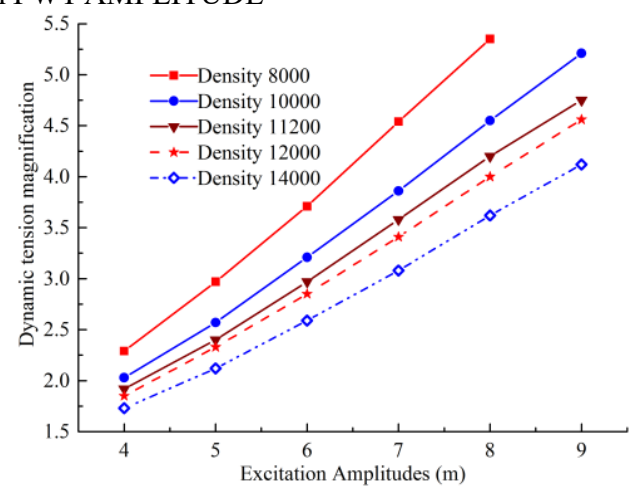

FIGURE 18. TENSION MAGNIFICATION AT DIFFIERENT MASS DENSITIES 
Initial tension is one of important design factors by which initial angle and shape of the catenary can be determined statically, then the top tension and restoring performance would be directly influenced during dynamic response, as shown in Fig.1 and Eq.(8). The dynamic response of the catenary with different values of initial tension ratio (the top tension to the structural gravity) and the corresponding initial shape (indicated as the horizontal projection), as listed in Table 2, are analyzed so as to examine the impact of the initial tension.

The RMS displacements along the catenary length, at FWT's $0.1 \mathrm{~Hz}$ frequency and $6 \mathrm{~m}$ amplitude, are plotted in Fig.19. As the tension ratio gets larger, the RMS displacement gets larger while the slack gets more likely to happen. Principally because the traits of catenary which is initially shaped by the top tension. As a comparison, the RMS displacements of quasi-static method is also presented in Fig.20. Comparing Fig.19 and 20, we can see that, at same values of FWT's amplitude and frequency, as the tension ratio rises the displacement gets larger, or velocity gets larger and consequently the dynamic tension is larger. Figure 21 shows the tension amplification (the ratio of the maximum dynamic tension to the static one) against the initial tension, where the tension amplification rises linearly with the increase of the initial tension. It means the impact of FWT's amplitude on tension amplification becomes more profound as the initial tension ratio rises.

TABLE 2. THE PARAMETERS OF THE MOORING-LINE

\begin{tabular}{ccc}
\hline Case & Horizontal projection $/ \mathrm{m}$ & Initial tension ratio \\
\hline 1 & 700 & 1.52 \\
2 & 701 & 1.55 \\
3 & 702 & 1.58 \\
4 & 703 & 1.62 \\
5 & 704 & 1.66 \\
6 & 705 & 1.71 \\
7 & 706 & 1.76 \\
8 & 707 & 1.82 \\
9 & 708 & 1.88 \\
10 & 709 & 1.96 \\
\hline
\end{tabular}

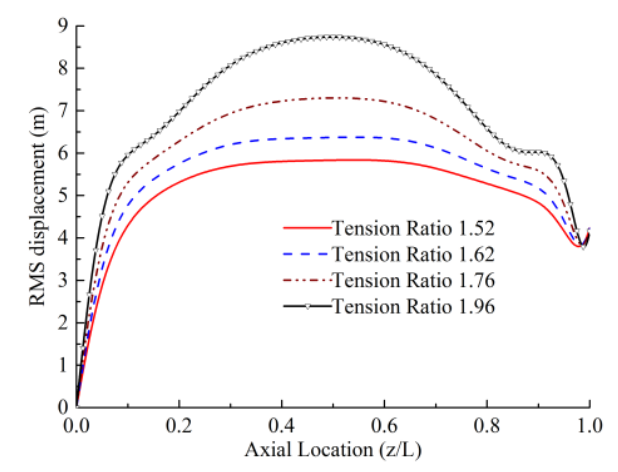

FIGURE 19. RMS DISPLACEMENT AT DYNAMIC CONDITION

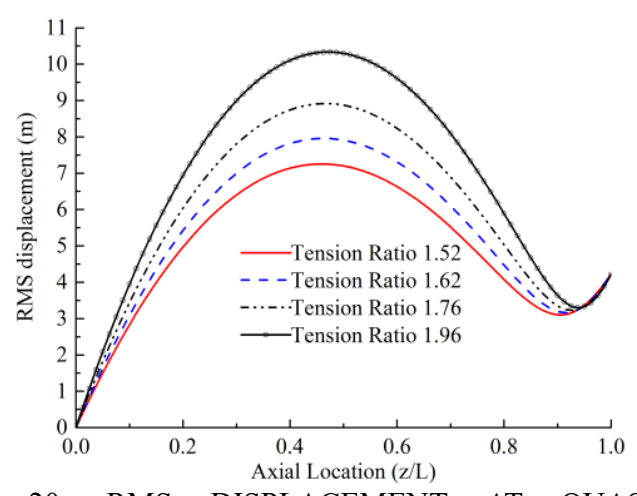

FIGURE 20. RMS DISPLACEMENT AT QUASI-STATIC CONDITION

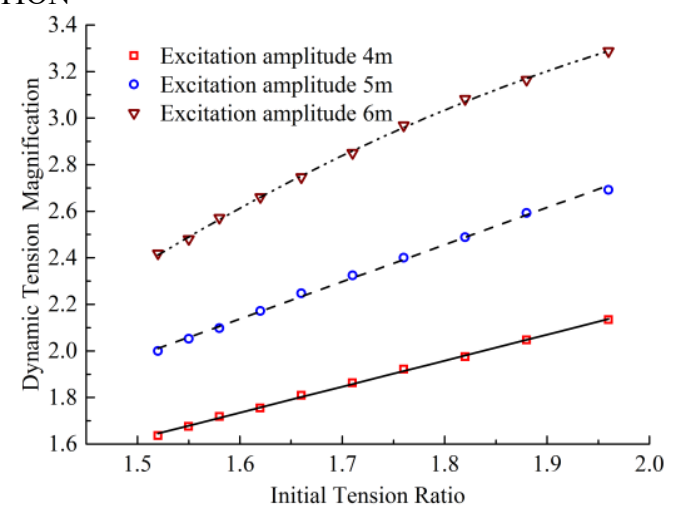

FIGURE 21. THE TENSION MAGNIFICATION VERSUS THE INITIAL TENSION RATIO

\section{CONCLUSION}

The restoring performance of the catenary mooring-line is examined while the dynamic behavior of the catenary mooringline, i.e. the structural/hydrodynamic inertia and damping effects, is included, compared to the previous quasi-static method. First, based on the 3D curved-flexible beam equations and the modified FEM numerical simulations, the nonlinear dynamic response model of the mooring-line is developed. Then the influences of the amplitude and frequency of FWT's motion, along with mooring-line's structural parameters, on the catenary's displacement and dynamic tension are studied and compared with the quasi-static results. Also, the slack-taut phenomenon and the snap tension during slack-taut owing to the dynamic effects, i.e. the structural gravity being balanced by the inertial/damping forces and the top tension being zero, are analyzed. Based our numerical results we draw the following conclusion:

1) When the motion of the FWT is smaller, the dynamic response of the mooring-line is characterized as a stable stand wave. The value of additional dynamic tension due to dynamic effects is about $20 \%$ of the quasi-static method, and the tension difference between the peak and trough is around three times of quasi-static method.

2) As FWT's motion gets larger, the slack is more likely to happen and the dynamic tension, at the excitation frequency, is about $30 \%$ higher than the quasi-static tension. When the 
mooring-line undergoes slack-taut, the response is characterized as travelling wave. The maximum dynamic tension gets larger up to 9 times of the quasi-static value. And, the catenary velocity at higher frequencies get larger too, e.g. the tension at twice excitation frequency even becomes bigger than that at excitation frequency. The higher tension at higher frequency should be paid attention during structure safety assessment because it is much crucial to fatigue life of catenary.

3 ) The influences of mooring-line's mass density and/or initial tension on the dynamic top tension, or the magnification factor of top tension caused by mooring-line's dynamic behavior, become more profound with the decrease of mass density or increase of the initial tension ratio.

\section{ACKNOWLEDGMENTS}

The authors of this paper would like to thank the financial supports provided by the National Natural Sciences Foundation (Grant No. 11232012 and 11372320).

\section{REFERENCES}

[1] Musial, Walt, Sandy Butterfield, and Andrew Boone. "Feasibility of floating platform systems for wind turbines." 23rd ASME Wind Energy Symposium, Reno, NV. 2004.

[2] Yong-Pyo, Hong, et al. "An experimental study on the extreme motion responses of a spar platform in the heave resonant waves." The Fifteenth International Offshore and Polar Engineering Conference. International Society of Offshore and Polar Engineers, 2005.

[3] Sarkar A, Eatock Taylor R. Dynamics of mooring cables in random seas[J]. Journal of Fluids and Structures, 2002, 16(2):

193-212.

[4] Mavrakos S A, Chatjigeorgiou J. Dynamic behaviour of deep water mooring lines with submerged buoys [J]. Computers \& structures, 1997, 64(1): 819-835.

[5] Papazoglou V J, Mavrakos S A, Triantafyllou M S. Nonlinear cable response and model testing in water $[\mathrm{J}]$. Journal of sound and vibration, 1990, 140(1): 103-115.

[6] Van Den Boom, H.J.J. Dynamic behaviour of mooring lines. Proceedings of Behaviour of Offshore Structures, 1985:359368.

[7] Gobat J I, Grosenbaugh M A. A simple model for heaveinduced dynamic tension in catenary moorings[J]. Applied Ocean Research. 2001, 23(3): 159-174.

[8] Qiao Dongsheng, Ou Jinping. Static analysis of a deepwater catenary mooring system, Ship\&Ocean Engineering, 2009, Vol.38(2):120-124.

[9] Zhang Huoming, Zhang Xiaofei, Yang Jianmin, Static characteristic analysis of multi-component mooring line based on optimization thinking, Ship Science and Technology, 2010. Vol.32(10): 114-121

[10] Vassalos D, Huang S. Dynamics of small-sagged slack-taut marine cables [J]. Computers \& structures, 1996, 58(3): 557562.
[11] Huang S, Vassalos D. A numerical method for predicting snap loading of marine cables [J]. Applied Ocean Research, 1993, 15(4): 235-242.

[12] Chen X, Zhang J, Ma W. On dynamic coupling effects between a spar and its mooring lines $[\mathrm{J}]$. Ocean Engineering. 2001, 28(7): 863-887.

[13] Zhang S, Tang Y, Liu X. Experimental investigation of nonlinear dynamic tension in mooring lines $[\mathrm{J}]$. Journal of Marine Science and Technology. 2012, 17(2): 181-186.

[14] Zhang S, Tang Y, Liu X. Analysis of Nonlinear Dynamic Response of Mooring Lines in Deepwater[C]. ASME 2010 29th International Conference on Ocean, Offshore and Arctic Engineering. American Society of Mechanical Engineers, 2010: 489-494.

[15] Lindahl J, Sjoberg A. Dynamic analysis of mooring cables, Proceedings of second international symposium on ocean engineering and ship handling, Sweden, 1983:281-319.

[16] Paulling J R, Webster, W C. A consistent large-amplitude analysis of the coupled response of a TLP and tendon system. Proceedings of Offshore Mechanics and Arctic Engineering, OMAE, Tokyo, Japan, 1986, 3:126-133.

[17] Kwan C T, Bruen F J. Mooring Line Dynamics: Comparison of Time Domain, Frequency Domain, Quasi-static Analyses. Proceeding of the 23rd Offshore Technology Conference: Houston TX, May 1991, 95-108.

[18] Chen X. Studies on dynamic interaction between deepwater floating structures and their mooring/tendon systems [D]. Texas A\&M University, 2002.

[19] Love A E H. A treatise on the mathematical theory of elasticity [M]. Cambridge University Press, 2013.

[20] Garrett D L. Dynamic analysis of slender rods [J]. Journal of energy resources technology. 1982, 104(4): 302-306. 